\title{
Bemerkungen zu meiner Abhandlung "Über die im weiblichen Geschlechte ungeflügelte und schwingerlose Dipterengattung Epidapus Hal.".
}

\author{
Von Karl Czǐ̌ek, Brünn.
}

(Mit 3 Figuren.)

$\mathrm{Zu}$ der oben zitierten, in der Wiener Entomol. Zeitung Jahrg. XXXIV, 1915, erschienenen Abhandlung über die Gattung Epidapus Hal. sind einige teils ergänzende, teils berichtigende $\mathrm{Zu}$ sätze notwendig geworden, die ich zum Teil bereits in den „Beiträgen zur rezenten Fauna der mährischen Höhlen“ (Zeitschrift des Mährischen Landesmuseums, Brünn, XV. Bd., 1916 S. 32) gebracht habe. Da aber auch durch die dort niedergelegten Bemerkungen die ganze Frage nicht vollständig geklärt wurde, halte ich es für notwendig, auf meinen Artikel vom Jahre 1915 nochmals zurückzukommen.

Bei der Abfassung meiner Arbeit über Epidapus konnte ich die wichtige Arbeit Enderleins: „Die phyletischen Beziehungen der Lycoriiden zu den Fungivoriden und Itonididen und ihre systematische Gliederung“ im Archiv für Naturgeschichte, 77. Jahrg. 1911, 3. Suppl. nicht benützen. Erst nach Drucklegung meiner Abhandlung war es möglich, in die Arbeit Enderleins Einsicht zu nehmen, und ich mußte erkennen, daß die schematischen Abbildungen der männlichen und weiblichen Tiere, die ich in meiner Arbeit gebracht hatte, Zweifel an der Zugehörigkeit der beiden beschriebenen Arten zu der Familie der Sciaridae (Lycoriidae) hervorrufen müßten. In den Abbildungen - besonders fällt dies bei Fig. 5 in der Abbildung des Weibchens auf - erscheinen die Augen auf der Stirne durch einen breiten Raum getrennt, was der Augenbildung der Sciaridae nicht entsprechen würde. Nach Enderlein besitzen ,alle Lycoriiden an jedem Auge einen schmalen Fortsatz; diese Fortsätze beider Augen laufen hinter der Fühlerbasis und vor den Ozellen über die Stirne und stoßen in der Mitte zusammen oder nähern sich wenigstens so stark, daß nur ein ganz winziger Zwischenraum die beiden Enden trennt". Enderlein bezeichnet diese schmale Augenverbindung mit "A ugenbrücke“.

Bei der ersten Untersuchung der Tiere hatte ich der Augenbildung nicht die notwendige Aufmerksamkeit geschenkt; durch Herrn 
Prof. H. Schmitz auf den Widerspruch aufmerksam gemacht, wurden die Tiere, die ich als Epidapus atomarius beschrieben hatte, einer neuerlichen Untersuchung unterzogen. Die in Alkohol konservierten Stücke erwiesen sich für die Untersuchung unbrauchbar, da die Konturen der Augen nicht scharf hervortraten; die Stücke wurden daher in Terpentin aufgehellt und nun konnte bei beiden Ge-

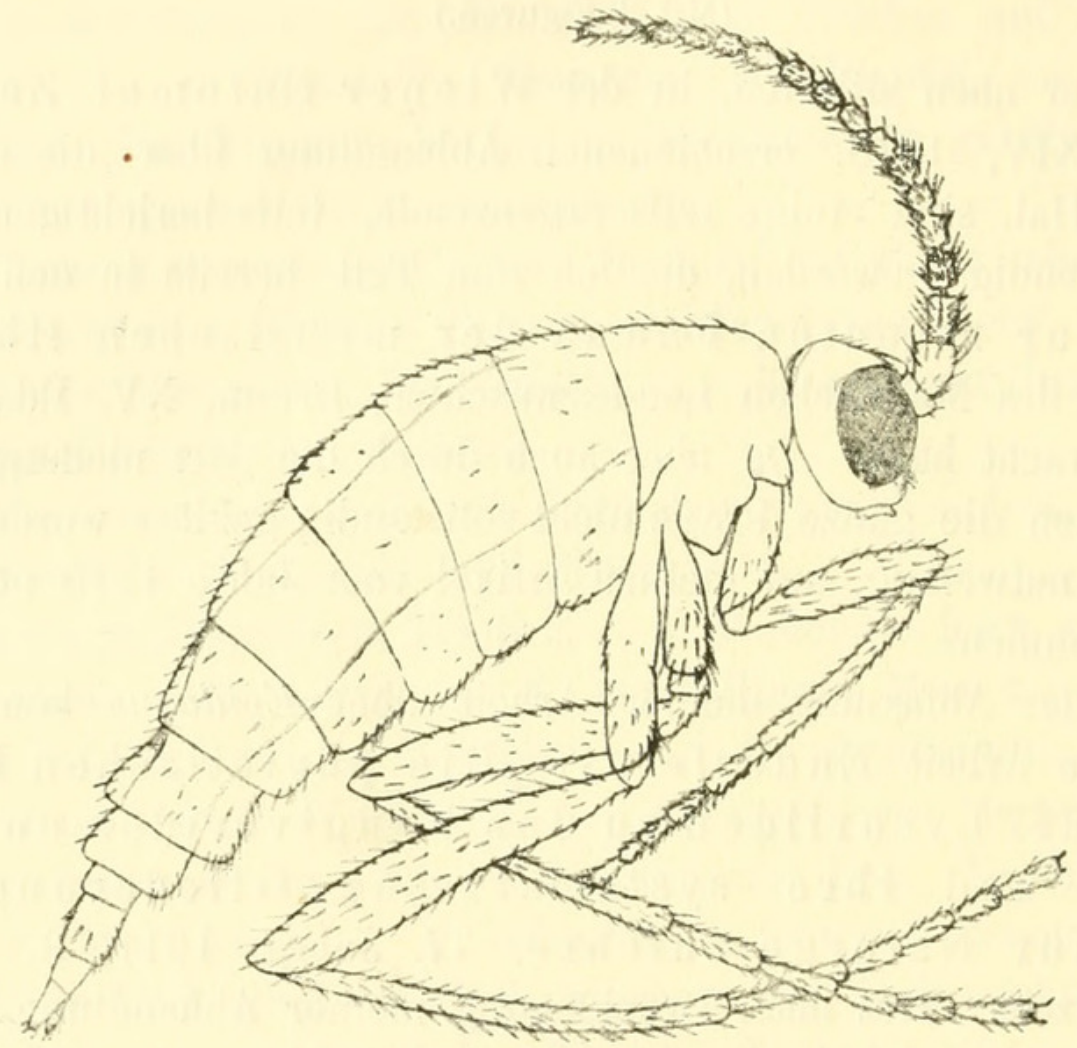

Fig. 1. Epidapus atomarius Dg. ‥

schlechtern von Epidapus atomarius und bei Epidapus Absoloni mihi die "Augenbrïcke" Enderleins genau und alle Zweifel ausschließend festgestellt werden. (Vgl. Fig. 2 u. 3.)

Damit erscheint die Zugehörigkeit der beiden Arten zu der Gruppe der Zygophthalmen Enderlein (Sciaridae, Scatopsidae und Cecidomyid(ce) sichergestellt. Die Dreizahl der Ozellen und das Vorhandensein der Media verweisen die Arten in die Familie der Sciaridae (Lycoriidae) im Sinne Enderleins. Innerhalb dieser Familie gehören sie zur Subfamilie Sciarinae (Lycoriinae) und zur Tribus Sciarini (Lycoriini).

Es bleibt aber noch eine Frage offen, die Frage, ob die in der mährischen Wy pustekhöhle gefundene, im weiblichen Geschlechte ungeflügelte Art, die ich als Epidapus atomarius angesprochen habe, und die von Dr. Absolon im Mährischen Karst entdeckte $E$. 
Absoloni mihi wirklich zur Gattung Epidapus gehören. H. Schmitz, dem ich einige Exemplare zur Untersuchung sandte, verwies darauf, daß Schiner ${ }^{1}$ ), Winnertz ${ }^{2}$ ) und van der Wulp ${ }^{3}$ ) der Halidayschen Gattung Epidapus viergliedrige Taster zuschreiben. Die Fliege aber, die ich als Epidapus atomarius beschrieben habe, besitzt, wie eine genaue Prüfung an vielen Exemplaren und in beiden Geschlechtern ergab, ${ }^{4}$ ) einen eingliedrigen Maxillarpalpus (den Trochanter ausgenommen); der Maxillarpalpus ist oval, trägt an der Außenseite eine längere Borste und apikal mehrere, hyaline

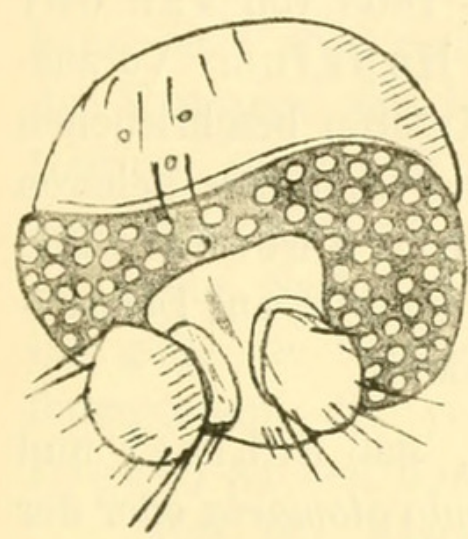

Fig. 2. Epidapus atomarius

Dg. ‥ Kopf von oben.

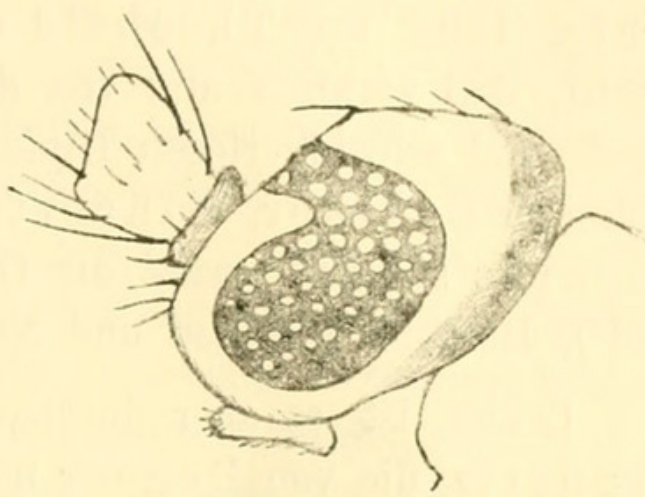

Fig. 3. Epidapus atomarius Dg. ㅇ. . Kopf von der Seite.

stäbchenförmige Anhänge, die als Sinnesorgane gedeutet werden könnten. Von der Seite gesehen, erscheint er ungefähr von der Form, wie ihn Enderlein für seine Art Ceratiosciara corniculata ${ }^{5}$ ) 우 abbildet. Damit tritt die "Epidapus-Frage" in ein neues Stadium.

Bis zum Jahre 1900 war Tipula atomaria Deg. (=Epidapus venaticus Hal.) die einzige bekannte Fliege ohne Flügel und ohne Schwinger, bekannt nur im weiblichen Geschlechte.

Chionea Dalm. 1816 $)$ ist flügellos in beiden Geschlechtern, besitzt aber deutliche Schwinger; Wasmanniella Kieffer 18987) ist zwar im weiblichen Geschlechte flügellos, besitzt aber rudimentäre Schwinger und ihre Larve ist gallenbildend.

1) Fauna austriaca, Die Fliegen. II, 416, 1864.

$\left.{ }^{2}\right)$ Beiträge zu einer Monographie der Sciarinen. Wien 1867, 182.

$\left.{ }^{3}\right)$ Tijdschr. voor Entomol., XI, 1868, 224.

4) Daß die untersuchten Männchen und Weibchen zusammengehören, unterliegt keinem Zweifel. Ich habe seither aus faulem Holze, das derselben Fundstelle entnommen war, zahlreiche $\sigma^{\alpha} \sigma^{>}$und $q$ 우 bis in den Okıber hinein gezogen.

5) Archiv für Naturgesch, 77. Jg., 1911, 1. Bd., 3. Suppl.

$\left.{ }^{6}\right)$ K. Vetensk. Acad. Handling. 102, 1816.

$\left.{ }^{7}\right)$ Bull. de la Soc. d'Hist. Natur. du Metz. S. 46.

Wiener Entomologische Zeitung, XXXVI. Jahrg., Heft IX-X (30. Dez, 1917). 
Von Monardia van der Wulpi Meijere $1899^{1}$ ) kommen neben Weibchen mit normalen, auch solche mit verkümmerten und Weibchen ohne Flügel vor; die Art besitzt aber (mikroskopisch kleine) Schwingkölbchen und gehört zu den Cecidomyiden. Palpus viergliedrig.

Die verborgene Lebensweise wohl aller flügellosen Dipteren hat es mit sich gebracht, daß flügel- und schwingerlose Zweiflügler von den Autoren sehr selten beschrieben bzw. erwähnt werden: 1778 von Degeer²), 1837 von Haliday ${ }^{3}$ ), 1864 von Schiner, 1866 von H. Loew ${ }^{4}$ ), 1867 von Winnertz, 1868 von van der Wulp, 1892 von Theobald und 1895 von Hopkins. Vorausgesetzt, daß wirklich alle von den erwähnten Autoren beschriebenen flügel- und schwingerlosen Dipteren zur Gattung Epidapus gehören (wohin sie Schiner, Winnertz, van der Wulp, Theobald und Hopkins stellten), wäre die Gattung aus der Schweiz, aus Deutschland ${ }^{5}$ ), Holland, England und Nordamerika bekannt.

Leider ist es aber durchaus nicht sicher, daß Schiner und Winnertz die von Dege er beschriebene Tipula atomaria oder der von Haliday entdeckte Epidapus venaticus vorgelegen ist. Erst Schiner und Winnertz haben auf Grund ihrer Untersuchungen dem Weibchen der von ihnen beschriebenen flügellosen Art viergliedrige Taster zugesprochen und ihre Art zu der Gattung Epidapus Hal. gestellt, bzw. als Epidapus venaticus angesprochen ${ }^{6}$ ). Haliday erwähnt aber in seiner kurzen Gattungsdiagnose, die mir nur aus L o e w (Schriften der Naturforsch. Gesellsch. in Danzig, Neue Folge,

1) Tijdschr. voor Entomol., 42. Bd. 1899 (1900), S. $140 \mathrm{ff}$.

$\left.{ }^{2}\right)$ Mém. pour serv. à l'hist. des Insectes. T. VII, 602, 1778.

3) Walker, Ins. Brit. Dipt. I, 7, 1851.

$\left.{ }^{4}\right)$ Schriften, Naturf. Ges. in Danzig. N. F. 1. Bd., 1866, 3. u. 4. Heft.

$\left.{ }^{5}\right)$ In den Arb. u. Veränd. der Schles, Ges. für vaterländ. Kultur im Jahre 1826, Breslau 1827, fand ich überdies in den Berichten noch folgende kleine Notiz: „Herr Schilling zeigte einige um Breslau gefundene Exemplare einer unbestimmten flügellosen Art." (Dipt.)

$\left.{ }^{6}\right)$ Ich kann nicht unerwähnt lassen, daß selbst die Beschreibungen Schiners und Winnertz' nicht ganz miteinander übereinstimmen, obwohl beiden Autoren zweifellos eine und dieselbe Art vorlag, da Schiner das von ihm untersuchte Exemplar nach seiner eigenen Aussage von Winnertz erhielt. Schiner bemerkt nämlich, daß die Vorderschenkel des Weibchens am breitesten seien und daß die Haftläppchen fehlen, Winnertz erwähnt das erste Merkmal überhaupt nicht, von den Pulvillen aber sagt er, daß sie mikroskopisch klein seien. 
I, 1866) bekannt ist, die Taster gar nicht und auch Degeer ${ }^{1}$ ) spricht mit keinem Wort von den Palpen. Er sagt vielmehr ausdrücklich: „Weil das Tier so klein war, konnte ich das Maul mit den Anhängen nicht recht untersuchen."

Ich habe nun a. a. O. die Vermutung ausgesprochen, daß Schiner und Winnertz getrocknete Stücke untersucht haben und gemeint, daß es dann bei der Kleinheit der Tiere ausgeschlossen ist, die Zahl der Tasterglieder genau zu bestimmen, da bei den getrockneten Exemplaren schon die Fühler derart zusammenschrumpfen, daß die Anzahl der Geißelglieder nicht mit Sicherheit anzugeben ist. Ich kann mir aber nicht gut denken, daf der so genaue Winnertz, der Tausende von Flügeluntersuchungen anstellte, seiner flügellosen Gattung auf Grund einer oberflächlichen Untersuchung dieselbe Zahl von Tastergliedern zugesprochen hätte wie der Gattung Sciara, wenn er seiner Sache nicht sicher gewesen wäre, noch dazu, da es für ihn galt, die strittige systematische Stellung der Gattung Epidapus festzustellen, die von Macquardta ${ }^{2}$ zu den Cecidomyiden, von Bigot ${ }^{3}$ ) zu den Tipuliden (Chioneen) gestellt worden war.

Um mir Klarheit zu verschaffen, wandte ich mich an Herrn Kustos A. Handlirsch mit der Bitte, mir die Typen der Winnertzschen Gattung, die ich im k. k. Naturhistor. Hofmuseum in Wien vermutete, zur Untersuchung zu überlassen, erhielt aber von dem Vorstand der dipterologischen Sammlungen, Herrn Dr. H. Z erny, die Mitteilung, daß sich die Typen von Exidapus Winn. nicht in den Wiener Sammlungen befänden. Mit der gleichen Bitte wandte ich mich dann an die zoologischen Sammlungen der Universität Bonn, in deren Besitz ein großer Teil der Koll. Winnertz übergegangen sein soll, habe aber bisher keine Nachricht erhalten.

Gelingt es, die Typen Winnertz' aufzufinden, und ergibt eine genaue Prüfung, daß die von ihm beschriebenen Tiere wirklich viergliedrige Taster besitzen, dann wird sich feststellen lassen, was für ein Zweiflügler von Schin er und W innertz unter dem Namen Epidapus beschrieben wurde. Wir werden dann aber leider noch immer nicht feststellen können, ob die Gattung Epidapus Winn. mit Tipula atomaria Deg. oder mit Epidapus venaticus Hal. zusammenfalle. Denn es ist seit dem Jahre 1900 eine Reihe von weiteren Dipteren beschrieben worden, die im weiblichen Geschlechte flügel- und

1) Degeers Abhandlungen zur Geschichte der Insekten, aus dem Fran zösischen übersetzt von Joh. Aug. Ephr. Goeze, Nürnberg 1783.

$\left.{ }^{2}\right)$ Hist. naturelle des Insectes. Dipt. I, 119, 1834.

$\left.{ }^{3}\right)$ Annal. Soc. Entomol. de Fr., Ser. III, T. II, ${ }_{1}^{4} 454,1854$.

Wiener Entomologische Zeitung, XXXVI. Jahrg., Heft IZ-X (30. Dez. 1917). 
schwingerlos sind und auf welche die Beschreibungen Degeers und Halidays ebenfalls angewendet werden könnten.

Da es nun nicht ausgeschlossen war, daß die von mir entdeckte Art $\mathrm{zu}$ einer der in jüngerer Zeit von Börner, Enderlein, Kieffer und Schmitz aufgestellten Gattungen der Sciariden gehöre, habe ich die in Enderleins vorhin zitierter Arbeit beschriebenen und die von Schmitz aufgestellten Gattungen mit meiner Art verglichen, wobei auch jene Gattungen mitberücksichtigt wurden, die zwar im weiblichen Geschlechte geflügeit sind, aber einen eingliedrigen Maxillarpalpus besitzen.

Von diesen Arten sind im vorhinein auszuscheiden die Gattung Allostoomma Schmitz 1915 ${ }^{1}$ ) und Dahlica larviformis End. (Corynoscelis eximia o Dahl olim), da bei beiden Arten die Augen keine "Augenbrücke" bilden.

Von den anderen Arten kämen zunächst hier in Betracht Plastosciar ${ }^{2}$ ) Bergr. 1899 (nach En d erle in = Pseudosciara Kieff. ${ }^{3}$ ) 1898) aus Zentraleuropa und Ceratiosciara ${ }^{4}$ ) Enderlein 1911 von den Seychellen, zwei Gattungen mit unbehaarten Augen. Erstere besitzt aber einen zweigliedrigen Maxillarpalpus, bei letzterer, die auch die Form des eingliedrigen Palpus mit meiner Art gemeinsam hat, wird als Gattungsmerkmal angegeben, daß die Fühler ungewöhnlich kurz seien, sie erreichen nur ein Viertel der Körperlänge und die Geißelglieder sind nur halb so lang wie breit.

Dasysciara $^{5}$ ) Kieff. 1903, Mycosciara6) Kieff. 1903 und Aptanogyna ${ }^{7}$ ) Börn. 1903 (letztere nach Enderlein synon. mit Peyerimhoffia Kieff. 1903) haben behaarte Augen, während ich bei meiner Art selbst bei starker Vergrößerung keine Pubeszenz der Augen entdecken konnte.

Dasysciara besitzt außerdem einen deutlich zweigliedrigen Palpus, die Vordertibien sind an der Spitze mit einer kammartigen Querreihe von Dörnchen besetzt, die Flügel des $q$ der einzigen bekannten Art (pedestris aus Südfrankreich) sind halb so lang wie der Thorax.

1) Tijdschr. voor Entomol., Deel LVIII, 289, 1915.

$\left.{ }^{2}\right)$ Mus. Nac. Buenos Aires I, 1899, 78.

$\left.{ }^{3}\right)$ Bull. Soc. Entom. de Fr., 1898.

4) Archiv für Naturgesch., 77. Jahrg., 1911, I, 3. Suppl.

$\left.{ }^{5}\right)$ Annal. Soc. Sc., Bruxelles, T. 27, 1903, 199.

$\left.{ }^{6}\right)$ ibidem, S. 203.

$\left.{ }^{7}\right)$ Zool. Anz., 26. Bd. 1903, 505. 
Mycosciara mit der einzigen bekannten Art brevipalpis Kiefi. aus Lothringen käme meiner Art nahe, der Palpus ist eingliedrig, das Empodium reduziert, Flügel $\sigma^{\top}$ ( $Q$ unbekannt) wie bei Sciara; aber die Augen sind behaart und die Härchen zwischen den einfachen Klauen sind bei meiner Art nicht so gestaltet, daf man sagen könnte, das Haftläppchen sei „hirschhornartig verzweigt".

Aptanogyna mit den Arten microthorax Börn. 1903 aus Sizilien, brachyptera Kieff. 1903 aus Südfrankreich, Schillei $\left.{ }^{1}\right)$ Börn. 1903 aus Galizien und aptera Kieff, 1903 aus Südfrankreich; $ㅇ$ 우 aller Arten ungeflügelt, von brachyptera auch das geflügelte $\sigma^{\top}$ bekannt. Als Gattungsmerkmal gibt Enderlein l. c. an: Palpus o zweigliedrig, des $Q$ einǵliedrig. - Da der Maxillarpalpus des o' meiner Art ebenfalls eingliedrig ist und sich auch in der Gestalt von dem des $q$ nicht unterscheidet, halte ich es für ausgeschlossen, daß meine Art in diese Gattung gehört oder mit einer der beschriebenen Arten zusammenfällt.

Es verbliebe noch die Gattung Pholeosciara ${ }^{2}$ ) Schmitz 1915 mit der Art mellina aus der Provinz Limburg in Holland, von J. Heselhaus in einem wahrscheinlich bewohnten Dachsbau aufgefunden. Nach der sehr genauen Beschreibung zu schließen, scheinen die von mir als Epidapus atomarius beschriebenen Tiere neben Mycosciara Kieff. der Gattung Pholeosciara Schmitz am nächsten zu stehen; ich kann sie aber nach meinen Untersuchungen, die von Kollegen Landrock nachgeprüft und bestätigt wurden, in diese Gattung nicht einreihen und mit der beschriebenen Art nicht identifizieren. Der Palpus meiner Art ist zwar eingliedrig wie bei Pholeosciara, auch die Stirn ist in einen schwachen nasenartigen Höcker vorgezogen und die Form des Prätarsus (die einfachen Klauen, die spärlichen "Fußbörstchen" von Empodium und Pulvillen) hat meine Art mit der Schmitzschen Gattung wohl gemein; dagegen ist der Maxillarpalpus meiner Art nicht mit Sinneshaaren, sondern mit hyalinen stäbchenförmigen Fortsätzen besetzt, die Augen sind nicht pubeszent, die Tibialsporne nicht behaart und an den Geißelgliedern der Fühler kann ich jene hyalinen, fadenförmigen Anhänge, wie sie Schmitz für seine Art angibt, nicht entdecken.

Die Beschreibung der von mir als Epidapus atomarius angesprochenen Art ist demnach durch folgende Kennzeichen zu erweitern :

1) Schmitz vermutet, daß diese Art Börners zu seiner Gattung Pholeosciara gehört.

$\left.{ }^{2}\right)$ Tijdschr. voor Entomol., Deel. LVIII, 1915, 285.

Wiener Entomologisehe Zeitung, XXXVI. Jahrg., Heft IX-X (30. Der. 1917). 19 
Maxillarpalpus bei $\sigma^{2}$ und $q$ eingliedrig, oval, an der Außenseite mit einer längeren Borste, apikal mit mehreren hyalinen Fortsätzen besetzt; Fühler $2+14$ gliedrig, Geißelglieder $\sigma^{\prime}$ lang, die des o kürzer gestielt, mit Wirtelbörstchen besetzt, die aber nicht regelmäßig (quirlförmig) angeordnet sind und ohne hyaline Anhänge. Stirn mit Augenbrücke, in der Mitte in einen kleinen stumpfen Höcker vorgezogen; Augen nicht behaart, drei Ozellen. Schienen mit kleinen, nichtbehaarten Endspornen. Kla uen einfach, nicht gezähnt, Empodium und Pulvillen aus einfachen, spärlichen Härchen bestehend.

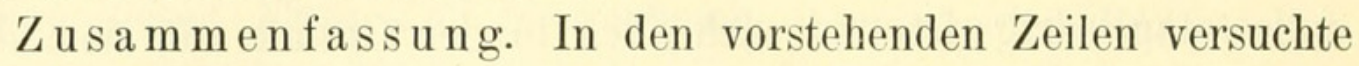
ich nachzuweisen, daß

1. die von Schiner und Winnertz als Epidapus venaticus bebeschriebene Art nicht mit Epidapus venaticus Hal. oder mit Tipula atomaria Deg. zusammenfallen $\mathrm{mu} B$;

2. daß die Beschreibungen Degeers und Halidays auch auf die in neuerer Zeit beschriebenen flügel- und schwingerlosen Dipteren angewendet werden können, daß es daher

3. heute unmöglich ist, die Arten Deegers und Halidays richtig zu deuten.

Sollte es sich später durch einen Vergleich mit den Typen W in nertz' erweisen, daß meine Art nicht zu der von ihm als Epidapus beschriebenen Gattung gehört, sollte sich durch Prüfung der Typen einwandfrei feststellen lassen, daß sie auch in keine der anderen bisher beschriebenen Gattungen eingereiht werden kann, sondern ein neues Genus bildet, so nenne ich die Gattung zu Ehren meines Freundes Karl Landrock, der sich durch seine Mycetophiliden-Arbeiten große Verdienste um die Kenntnis der Dipteren Mährens erworben hat, Landrockia n. g. und gebe ihr nach dem Lande, in dem sie zuerst entdeckt wurde, den Artnamen moravica $n$. sp.

Alle Zeichnungen wurden von Kollegen Karl Landrock ausgeführt. Die Klischees sind mir vom Kuratorium der Mähris chen Museumsgesellschaft in liberaler Weise für meine Arbeit zur Verfügung gestellt worden.

\section{Nachschrift.}

Herr Professor R. Hess e, Vorstand am Zoolog. und vergleich. anatom. Institut und Musum der königl. Universität Bonn, war so 
gütig, mir auf meine Bitte die von Winnertz in seiner Monographie der Sciarinen unter dem Namen Epidapus venaticus Hal. beschriebene Fliege zum Studium zu überlassen.

Mit anderen Arbeiten überbürdet, konnte ich meine Untersuchungen bisher noch nicht abschließen und bringe daher nur diese vorläufige Notiz als Nachschrift zu der vorstehenden Abhandlung.

Die mir übersandten Tiere aus der Sammlung Winnertz' sind relativ größer als die größten Exemplare meiner flügellosen Fliege aus den mährischen Höhlen. Sie zeigen im Bau der Fühler, der Beine und des Hinterleibes große Ähnlichkeit mit meiner unter dem Namen Epidapus beschriebenen Art, unterscheiden sich aber durch den auffallend stark und dicht behaarten Hinterleib, den fast ganz kahlen Rüickenschild, vor allem aber durch die deutlich vorragenden Maxillarpalpen, über deren Gestalt und Gliederung ich aber noch keine genauen Angaben machen kann, da die genadelten Tiere stark zusammengeschrumpft sind. Im Vergleich zu den winzig kleinen, kaum vorstehenden Maxillarpalpen meines "Epidapus" fallen die Mundteile der Tiere aus der Sammlung Winnertz' sofort durch ihre Größe auf.

Ich glaube daber schon heute mitteilen zu können, daß meine unter dem Namen Epidapus atomarius Deg. beschriebenen Tiere mit dem Winnertzschen Epidapus venaticus Hal. nicht zusammenfallen Eine ausführliche Beschreibung der Winnertzschen Tiere und eine Darstellung der ganzen Frage muß ich mir für spätere Zeit vorbehalten. 


\section{$2 \mathrm{BHL}$ Biodiversity Heritage Library}

Czižek, Karl. 1917. "Bemerkungen zu meiner Abhandlung "Über die im weiblichen Geschlechte ungeflügelte und schwingerlose Dipterengattung Epidapus HAL."." Wiener entomologische Zeitung 36, 283-291. https://doi.org/10.5962/bhl.part.12944.

View This Item Online: https://www.biodiversitylibrary.org/item/42783

DOI: https://doi.org/10.5962/bhl.part.12944

Permalink: https://www.biodiversitylibrary.org/partpdf/12944

\section{Holding Institution}

Smithsonian Libraries

\section{Sponsored by}

Smithsonian

\section{Copyright \& Reuse}

Copyright Status: NOT_IN_COPYRIGHT

This document was created from content at the Biodiversity Heritage Library, the world's largest open access digital library for biodiversity literature and archives. Visit BHL at https://www.biodiversitylibrary.org. 